\title{
Eberhard Stamm Frühbürgerliche Revolution und Recht in Deutschland
}

Reformation, Bauernkrieg und Rezeption des römischen Rechts: Von drei historischen Prozessen, die gleichsam synchron das 16. Jh. einleiteten und deren geschichtlicher Zusammenhang zumindest einer Frage wert wäre, fallen zwei in der gängigen Lehrbuch-Literatur zur Privatrechtsgeschichte der "Arbeitsteilung " idealistischer Wissenschaft zum Opfer. Da der Rechtshistoriker die Entwicklung des Rechts zu beschreiben und nicht in die Domäne des Historikers überzugreifen hat, scheint der deutsche Bauernkrieg grundsätzlich nur in einem kurzen Satz - im Rahmen globaler Hintergrundinformationen - gewürdigt werden zu dürfen: F. Wieacker' läßt an ihm (und an der "politischen Erhebung der Reichsritter «) den »Kampf um die Erneuerung der Verfassung des alten Reiches « scheitern, während Mitteis-Lieberich ${ }^{2}$ lakonisch konstatieren, daß er den Bauern keine Besserung ihrer Lage gebracht habe. Der Reformation immerhin wird etwas mehr Aufmerksamkeit geschenkt, wobei sie aber nur interessiert unter dem Gesichtspunkt des Rechts: "Auch die Reformation brachte nicht nur eine religiöse, sondern auch eine rechtliche Spaltung. « ${ }^{3}$

Interessant in diesem Zusammenhang erscheint mir die Tatsache, daß der Historiker (!) G. Franz in seinem Werk »Der deutsche Bauernkrieg " schreibt: »Der Bauernkrieg ist eine Auseinandersetzung zwischen genossenschaftlichem Volksrecht und dem obrigkeitlichen Herrschaftsrecht. « ${ }^{4}$ Tatsächlich ist auf fast jeder Seite des Buches von Recht, von alten und von göttlichem Recht, von Gerechtigkeit, Freiheit und Befreiung die Rede, wobei G. Franz die Vorstellungen der feudalen Gesellschaft über das Recht auf den ersten Seiten seines Buches durchaus treffend darstellt (Die Frage, wie und warum diese Vorstellungen entstanden sind, wird allerdings - in guter idealistischer Tradition - nicht gestellt.). Immerhin wird hier die Querverbindung zwischen Bauernkrieg und Rezeption gesucht ${ }^{5}$ und so der Ansatz einer komplexeren Betrachtungsweise ermöglicht.

Die Aufgabe dieser Arbeit wird es nun sein, auf dem Hintergrund der ökonomischen und sozialen Verhältnisse zu Beginn des 16 . Jhs. den bürgerlich-revolutionären Charakter von Reformation und Bauernkrieg, wie die materialistische Geschichtswissenschaft ihn herausgearbeitet hat, im einzelnen darzustellen, und im Anschluß daran die Rolle von Recht als Korrelat sozialökonomischer Verhältnisse am Beispiel dieses Zeitraumes, in dem sich der Ubergang von einer Produktionsweise zu einer anderen in revolutionären Bewegungen ankündigt, tendenziell aufzuzeigen.

\footnotetext{
1 Franz Wieacker, Privatrechtsgeschichte der Neuzeit, 2. Aufl. 1967, S. 133

2 Mitteis-Lieberidh, Deutsche Rechtsgeschichte, 10. Auflage 1966, S. 188.

3 Ders., a. a. O., S. 194.

4 G. Franz, Der Deutsche Bauernkrieg, 6. Aufl., Darmstadt i 962, S. 291.

5 Ders., a. a. O., S. 24 ff.
} 
Die Ergebnisse seien vorab kurz skizziert:

Gegen Ende des I s. und zu Beginn des 16. Jhs. spitzen sich die ökonomischen und gesellschaftlichen Widersprüche in Deutschland als Folge und Voraussetzung der Entwicklung von Keimformen kapitalistischer Produktion zur gesamtnationalen Krise zu. (S. I 31 ff.) Diese entlädt sich in den revolutionären Bewegungen der frühbürgerlichen Revolution, die ihren Höhepunkt in Reformation und Bauernkrieg findet. Progressive Kraft in dieser Zeit sind - sich ihrer Rolle aber nicht oder nur kaum bewußt - die kapitalistisch wirtschaftenden Teile des Bürgertums. Reaktionäre Kräfte sind eindeutig Klerus und Feudaladel. Territorialfürsten und Bauern verfolgen zwar subjektiv reaktionäre Ziele, dienen aber vielfach objektiv den progressiven Interessen des Bürgertums. (S. I $4 \circ \mathrm{ff}$.)

Unter diesem Gesichtspunkt ist auch die zur gleichen Zeit ihren Höhepunkt und Abschluß findende Rezeption römischer Rechtsformen zu sehen: Indem sie den subjektiven (reaktionären) Zielen der Territorialfürsten dienlich war, kam sie den objektiven (progressiven) Interessen des Bürgertums zugute. Mit Hilfe der Zentralgewalten führte die Rezeption zur Herausbildung einer bürgerlichen Juristenschicht und zu einer weitgehenden Umgestaltung in Gesetzgebung (Stadtund Landrechte), Verwaltung, Justiz (landesherrliche Gerichtszïge) und Rechtswissenschaft. (S. I 43 ff.)

Innerhalb der Letzteren spiegelt sich der Beginn der ökonomisch-gesellschaftlichen Umwälzung bereits in wissenschaftstheoretischen und methodologischen Auseinandersetzungen wider. (S. 146 ff.)

\section{Ökonomische Struktur}

In der 2. Hälfte des 15 . Jh. sprengte der sich bereits in den vorausgegangenen Jahrzehnten abzeichnende Aufschwung in der Entwicklung der Produktivkrätte allmählich die Schranken der einfachen Warenproduktion und führte zur Her-

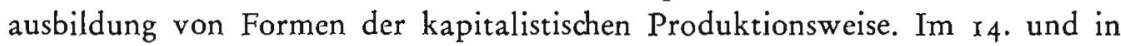
der ersten Hälfte des i 5 . Jhs. waren Elemente kapitalistischer Produktion nur sporadisch in den Städten Oberitaliens und der Niederlande aufgetreten.

Seit Anfang des 16. Jhs. aber breitete sich die neue Produktionsweise über große Gebiete Europas aus und siganlisierte den Beginn der kapitalistischen Ära.

In den ökonomisch fortgeschrittensten Ländern - England, Frankreich, Deutschland und den Niederlanden - schufen die Entwicklung der Produktionsinstrumente und die aus ihr resultierende Arbeitsteilung im Bergbau, Hüttenwesen, Schiffbau und in der Textilerzeugung Bedingungen für den Ubergang zur Manufaktur, die an der Schwelle des I6. Jhs. die höchste Form der gesellschaftlichen Produktion darstellte.

Besonders wirksam wurden die technischen Fortschritte im Hüttenwesen. Der Übergang vom Rennwerksbetrieb (Schmelze im offenen Kasten) zum Hochofenbetrieb erlaubte die Verhüttung auch schwer schmelzbarer Erze. Das Saigerverfahren ermöglichte die Trennung des Kupfers von anderen metallischen Bestandteilen und somit die Gewinnung reiner Metalle. Zum Betrieb von Hammerwerken und Blasebälgen wurden im zunehmenden Maß Wasserräder benutzt. Aus diesem Grunde verlagerte sich die Eisenindustrie von den Bergen und Wäldern in die Täler, wo sich in Manufakturen große Mengen von Arbeitern konzentrierten.

In der Textiltechnik erhöhte das um I 480 erstmals erwähnte Flügelspinnrad sowohl die Arbeitsproduktivität, als auch die Qualität der Produkte. 
Auf der Grundlage des um 1400 von Johannes Gutenberg in Mainz erfundenen Buchdrucks hatte sich ein neues bürgerliches Gewerbe gebildet. Es half, das kirchliche Bildungsmonopol zu brechen, und beeinflußte wesentlich die Entwicklung eines neuen, fortschrittlichen Denkens, sowie die Herausbildung einer antifeudalen öffentlichen Meinung.

Vor allem im Bergbau aber begann gegen Ende des i 5 . Jhs. ein stürmischer Aufschwung, der auf die gesamte Wirtschaft Deutschlands eine nachhaltige Wirkung ausübte. Unentbehrliche Voraussetzungen dafür waren vor allem die günstigen natürlichen Bedingungen Deutschlands:

Sachsen, Oberharz, Württemberg, Steiermark und Tirol waren im 15. und I6. Jh. außerordentlich reich an Erzvorkommen, besonders an Edelmetallen.

Ende des 15. und in der ersten Hälfte des I6. Jhs. nahm Deutschland in der Erzgewinnung in Europa den ersten Platz ein. So kamen ca. $80 \%$ der europäischen Silberproduktion aus den deutschen Ländern. ${ }^{6}$ Der Aufschwung in der Bergindustrie wurde hervorgerufen durch die Anforderungen des inneren und besonders des äußeren Marktes. Deutschland dominierte auf den bekannten Weltmärkten für Kupfer in Antwerpen und Leipzig, in Venedig, Frankfurt am Main und Nürnberg. Ein ununterbrochener Silberstrom floß aus Deutschland nach den großen europäischen Märkten Rom, Mailand, Venedig, Madrid, Sevilla, Saragossa, Lissabon, Antwerpen, Lyon, ganz zu schweigen von den Silbermärkten Deutschlands selbst.

Noch in der ersten Hälfte des I 5. Jh. wurde das Erz entweder durch einzelne Bergleute (Eigenlehner) oder durch Gruppen von Bergleuten gewonnen. Die Produktion lag also vor allem in den Händen kleiner selbständiger Produzenten, befand sich somit noch auf der Stufe der einfachen Warenproduktion. Ende der 7oer Jahre des I 5 . Jh. jedoch hörten diese kleinen Unternehmen auf, irgendeine bedeutende Rolle im Bergbau zu spielen.?

Die Gründe dafür liegen sowohl im ökonomischen, als auch im außerökonomischen Bereich: Die Ausdehnung der europäischen Handelsbeziehungen seit dem 12. Jh. ${ }^{8}$ war eine wichtige Voraussetzung für die Entfaltung der Warenproduktion in Deutschland gewesen. Das steigende Angebot fremder Waren hatte ständig neue Bedürfnisse - insbesondere des Adels ${ }^{\theta}$ und der vermögenden Bürgerschichten - hervorgerufen. Man begann, für die Ausfuhr zu produzieren, um sich Aquivalente zu verschaffen. Die Folge war eine enorme Steigerung der Produktivität - vor allem im Verlaufe des I5. Jh. -, die erreicht wurde durch die Schaffung neuer Produktionsinstrumente, durch die Verbesserung der Fertigungsme-

6 J. M. Grigor'jan, Aus der Geschıchte der Genesıs der kapitalistıschen Verhältnisse in Deutschland, in: Zeıtschrift für Geschichtswissenschaft (ZfG), I959, S. I745 ff.

7 Ders., a. a. O., S. 1749.

8 Als Bsp. hierfür möge der Levantehandel Venedigs und Genuas dienen: Seit dem Ir. Jh. wurde die arabische Mittelmeerherrschaft durch die Reconquista und die Kreuzzüge aufgelöst. Der Handel mit dem Westen fiel an Genua, Pisa, Neapel; der östlidhe Levantehandel an Sizilien und Venedig. Begehrte Orientwaren waren: Seide, Brokate, Damast- und Gazestoffe, Baumwolle, Kamelhaar, Elfenbein, Porzellan, Farbstoffe, Gewürze, Parfüme, Arzneımittel, Perlen, Edelsteıne. Sie gelangen über die von Arabern kontrollierten asıatıschen Karawanenwege (Seidenstraße) oder den Indischen-Ozean-Handel zur Levante und werden gegen Tuche und andere Erzeugnısse der europäischen Gewerbelandschaften (Mailand, Florenz, Oberdeutschland, Flandern, Brabant) gehandelt.

Die Stadtrepubliken Venedig und Genua gewinnen an Reichtum und Macht durch I. Transportgeschäfte während der Kreuzzüge; 2. stcigenden Geldbedarf (Bank- und Kreditgeschäfte); 3. byzantinische und arabische Handelserfahrungen (schriftliche und rationale Handelsmethoden); 4. durch den Zusammenbruch Siziliens und den Niedergang von Byzanz.

- Beim Adel manifestierte sich der wachsende Geldbedarf vorübergehend sogar darin, daß er z. T. auf die reale Ableistung von Frondiensten verzıchtete, und sıch diese in Geld auszahlen ließ. 
thoden und durch allmähliche Umgestaltung der Arbeitsorganisation. Das ganze I s. Jh. war also geprägt von einer ständigen Ausweitung der Warenproduktion und damit von einem steigenden Angebot nun nicht mehr vorwiegend ausländischer, sondern auch sehr viel mehr selbst produzierter Waren auf dem deutschen Markt. Die Folge war ein wiederum steigender Geldbedarf und eine stetige Steigerung des Wertes der Edelmetalle, die ja unmittelbar als Äquivalent dienten. Die daraus sich ergebende Notwendigkeit verstärkten Abbaus von Silber und Kupfer in Deutschland führte um I soo zu bedeutenden technischen Fortschritten in der Bergindustrie. Die in dieser Zeit gemachten Erfindungen und Vervollkommnungen hatten eine wesentliche Entwicklung der Produktivkräfte zur Folge.

Mit der Entwicklung der Bergbautechnik aber wurde das Vorhandensein von Kapital zur notwendigen Voraussetzung für die Produktion, da die erforderlichen Werkzeuge und Maschinen für die bisher handwerklichen Produzenten unerschwinglich wurden. Bei der Gründung neuer Bergbauunternehmen, die jetzt in zunehmendem Maße die Organisationsform der Manufaktur annahmen, spielten darum die bereits vorhandenen großen Geldvermögen der Handelskapitalisten eine große Rolle.

Besonders der deutsche Fernhandel hatte zur Zusammenballung bedeutender Geldfonds geführt. Kapitalkräftige Händler gründeten zu Ende des is. und zu Beginn des 16. Jh. große Handelsgesellschaften. Die bedeutendsten von ihnen befanden sich in Oberdeutschland, so die Fugger, Welser, Hochstetter, Paumgartner, Herwarth, Haug, Rehlinger, Manlich, Imhof und Tucher. Die Grundlage ihrer Vermögen war verschiedener Art: Bei den Rehlingern rührte es aus dem Verkauf adligen Grundbesitzes her; bei den Fuggern, die ursprünglich Barchentweber gewesen waren, aus gewerblichen Gewinnen, die sich durch Anwendung des Textilverlagssystems sprunghaft erhöht hatten. Aber so groß die Gewinne aus dem Fernhandel auch sein mochten, den raschesten Aufstieg erlebten jene Unternehmen, die sich der Geldspekulation und dem Edelmetallhandel zuwandten: Die Fugger und Paumgartner in Augsburg.

Im Verlauf von 80 Jahren war das Kapital der Fugger z. B. auf das siebzigfache angewachsen: 1464 betrug es $100000,-$ I 527 bereits 2,8 Millionen und I 546 sogar 7 Millionen Gulden. ${ }^{10}$

Neben den Fuggern und Paumgartnern taten sich besonders die Welser hervor. Einerseits beteiligten sie sich erfolgreich am Bergbau, andererseits drangen sie mit Gewinn in den Überseehandel ein. I 528 verpflichteten sie sich in einem Vertrag mit der spanischen Krone, den Sklavenhandel in Amerika zu organisieren.

Gleichzeitig übernahmen sie die Metallschmelze aus den Kolonien, brachten 30 deutsche Bergleute nach Amerika und gründeten in Venezuela eine deutsche Kolonie.

Bei der Art und Weise nun, in der das Handelskapital in den Bergbau eindrang, in der es also zu industriellem Kapital wurde, lassen sich drei Hauptwege unterscheiden: Entweder finanzierten die Kaufleute den Geldbedarf von Territorialherren, Kaiser und Papst (Hofhaltung, Verwaltung, Kriege, Kaiserwahlen, etc.. ..) und ließen sich zur Sicherung ihres Darlehens den Ertrag von Bergwerken zu niedrigem Preis übertragen, oder sie wandten das in der Textilindustrie bereits vorherrschende Verlagssystem an, oder sie beteiligten sich direkt mit $\mathrm{Ka}$ pitaleinlagen an Bergwerksunternehmen. ${ }^{11}$

10 Max Steinmetz, Lehrbuch der deutschen Geschıchte (Beıträge Nr. 3), Deutschland von 1476 bis 1648 , Berlin 1967, S. 18 .

11 Durch Pacht beim Fiskus, dem durch das Bergregal ein bestımmter Anteil der Ausbeute ge- 
Zu den Waren aber, die der Bergbauunternehmer nunmehr kaufen mußte, um auf höherer Stufe als der einfachen Warenproduktion produzieren zu können, gehörte außer den oben erwähnten Werkzeugen und Maschinen vor allem die menschliche Arbeitskraft. Darum waren die Arbeiter dieser Unternehmen bereits Lohnarbeiter, wie sich aus den Bergstatuten dieser Zeit entnehmen läßt. Die ubbliche Formel lautete hier: "Jeder Arbeiter muß wöchentlich Sonnabends persönlich beim Schichtmeister erscheinen, um seinen Arbeitslohn zu empfangen $« .1 \%$

Die Reserven der freien Arbeitskräfte rekrutierten sich zu einem großen Teil aus der Masse der Landbevölkerung. Teile der von der feudalen Ausbeutung unterdrückten Bauern verließen die Landwirtschaft und wanderten in die etwas freieren Bergbaubezirke ab, wo ihnen die staatliche Macht, die an der Ausweitung der Metallgewinnung sehr interessiert war, im Vergleich zu anderen Schichten der Bevölkerung bessere Lebensbedingungen bot, wie z. B. das Recht der Freizügigkeit, der unentgeltlichen Wald-, Weg- und Wassernutzung, Befreiung von einigen feudalen Abgaben und im Allgemeinen auch vom Kriegsdienst. Schließlich brauchten die geflüchteten Bauern, die als Bergleute arbeiteten, nicht zu ihren feudalen Landbesitzern zurückzukehren und hatten sich so von ihrer feudalen Abhängigkeit befreit. Hier werden bereits Tendenzen zur Untergrabung des Feudalismus deutlich.

Der Bergbau spielte sowohl ökonomisch, als auch politisch eine relativ große Rolle. Er hatte - politisch gesehen - eine erstrangige Bedeutung für das Kräfteverhältnis zwischen den verschiedenen fürstlichen Territorien. Durch den Bergbau wurden die großen Handelsgesellschaften zu enormen Geldaufwendungen in Form von Darlehen an die Fürsten (vgl. oben: Eindringen des Handelskapitals in den Bergbau) motiviert.

Ohne dieses Geld wäre eine Ausrüstung und Aufrechterhaltung der riesigen Söldnerheere für die nicht enden wollenden Kriege, wäre der starke Verwaltungsapparat der Fürsten und die Bestreitung ihrer gewaltigen Hofhaltungskosten nicht denkbar gewesen.

Okonomisch gesehen war der Bergbau ein wichtiger Träger progressiver Entwicklungen. Da Edelmetalle als allgemeines Äquivalent fungierten, wirkte sich die Ausweitung der Edelmetallproduktion belebend auf den gesamten Waren-

hörte, erhielt der Kaufmann oder Bankıer als Garantie für die dem Herrscher gegebenen Darlehen, das Recht des Aufkaufs der Metalle beim Bergwerksbesitzer zu einem unter dem Marktpreıs liegenden Preıs. In der Folge ging er dann selbst zum industriellen Unternehmen über - entweder durch den Kauf des Bergwerkes, durch Anteile daran, oder durch den unmittelbaren Aufbau neuer Unternehmungen. Dieser Weg war besonders charakteristisch für den Tiroler Bergbau. Der zweite Weg war das sog. Verlagssystem, d. h. die Gewährung von Geldmitteln an Bergbauunternehmer durch Kaufleute und das damıt verbundene Recht für Jetztere, das Metall zu günstigem Press zu kaufen. Messt wurden die Unternehmen dieser Bergbaubesitzer als Sicherheit für die gewährten Kredite verpfändet und gingen bei Zahlungsunfähigkeit des Schuldners in das Eigentum des Gläubigers über, der sich dann selbst als Unternehmer betätıgte.

Beım dritten Weg beteiligten sich die Kaufleute unmittelbar an den Gewerkschaften, die seit Ende des 15. Jhs. zu einer der wesentlichen Organisationsformen der Metallgewinnung in großem Ausmaß wurden. Beı diesem Weg sind von vornherein die Geldmittel der Kaufleute ohne jegliche vorhergehende Handelsoperatıon direkt für die Organısatıon der industricllen Produktion bestimmt. Das Eröffnungskapital der Gewerkschaft bestand aus der Summe der Eintrittsbeiträge ihrer Mitglieder und war gewöhnlich in 28 Anteile (Kuxe) aufgegliedert. Die Anzahl der Anteile jedes Mitgliedes hıng von seıner Kapitaleınlage ab. Gewinn und Produktionskosten wurden entsprechend der Anzahl der Anteile umgelegt. Teilhaber waren Fürsten, Ritter, Geistliche, meıstens aber große Kaufleute und Räte der Städte.

12 Das sächsısche Bergrecht des Mittelalters, hrsg. v. H. Ermısch, Leıpzig, 1887, S. 132: $\$ 62$ des Schreckenberger Bergstatuts. 
verkehr, insbesondere mittels des Geld- und Kreditgeschäfts auf den Außenhandel aus.

Die Geld-Ware-Beziehung intensivierte sich. Edelmetalle ließen sich unmittelbar in Geld umwandeln, das teils der feudalen Konsumtion zugute kam, teils aber auch in die Produktion zurückfloß.

So äußerte sich die Erweiterung der Reproduktion im Bergbau bereits in typisch kapitalistischer Weise, nämlich darin, daß ein Teil des angeeigneten Mehrwerts in tätiges Kapital verwandelt, also akkumuliert wurde. Der Edelmetallbergbau nahm dabei insofern noch eine besondere Position ein, als die Uberführung des Metallwerts aus der Waren- in die Geldform nicht wie sonst, durch den Verkauf vor sich ging, sondern unmittelbar im Produktionsprozeß selbst.

Aber nicht nur im Bergbau zeigen sich in dieser Zeit frühkapitalistische Elemente. Auch im Textilgewerbe, in der Holzverarbeitung und im Buchdruck, im Hüttengewerbe und in der Metallverarbeitung wurde der Rahmen der einfachen Warenproduktion gesprengt.

In all diesen Bereichen verlor der Handwerker durch das Verlagssystem Schritt für Schritt die Verfügungsgewalt über seine Produktionsmittel. Die Vertiefung der Ware-Geld-Beziehungen hatte allgemein einen höheren Geldbedarf zur Folge. Diesen konnte der Handwerker nicht mehr allein durch den Verkauf seiner Erzeugnisse decken und geriet so in den Einflußbereich der Handels- und Kreditfirmen. Durch Kredite und Rohstoffvorschüsse, durch Lieferverträge mit ganzen Zünften (Zunftkauf) wurden Handwerk und gewerbliche Produktion in eine sich allmählich vertiefende wirtschaftliche Abhängigkeit gebracht.

So zeigen sich in den verschiedenen Produktionszweigen vielfältige Frühformen kapitalistischer Produktion. Bei alledem muß aber berücksichtigt werden, daß sich diese Entwicklungen »im Schoße des Feudalismus «, also eingebettet in feudale Produktionsverhältnisse, vollzogen, die von der politischen Gewalt des Adels verteidigt wurden. So konnte der Frühkapitalismus in Deutschland manche feudale Fessel sprengen, konnte er die Widersprüche der alten Produktionsweise offen zutage treten lassen und Krisen schüren. Er konnte aber nicht die alte Produktionsweise völlig beseitigen. Nach wie vor herrschten - vor allem auf dem Lande - feudale Strukturen und Abhängigkeiten, wußten Adel und Klerus sich auf feudale Art und Weise das gesellschaftliche Mehrprodukt anzueignen, waren in den Städten die feudalen Zunftverfassungen ein ernstes Hindernis für die massenhafte Warenproduktion auf kapitalistischer Stufe.

Ebenso muß berücksichtigt werden, daß sich das Vorkommen kapitalistischer Elemente jeweils auf einige »Industriezentren « beschränkte, daß also nur einzelne Gebiete Deutschlands betroffen waren, die untereinander allerdings über große Fernstraßen regen Handel trieben.

Außerdem stellte die staatliche Zersplitterung insofern eine starke Fessel für die Ausweitung der kapitalistischen Produktion dar, als diese nicht - wie z. B. in England und Frankreich - auf die Gewährleistung eines nationalen Binnenmarktes und auf Schutz nach außen für den Außenhandel durch eine staatliche Zentralgewalt rechnen konnte.

Schließlich war Deutschland am Ende des I 5 . Jhs. ungeachtet des stürmischen Aufschwungs der gewerblichen Produktion überwiegend ein Agrarland. Etwa $75 \%$ der I $2-13_{3}$ Millionen Einwohner lebten auf dem Lande. Nach wie vor bildete der Antagonismus zwischen Bauern und Feudalherren den gesellschaftlichen Grundwiderspruch.

Der Entwicklungsstand der landwirtschaftlichen Produktivkräfte war in den verschiedenen Bereichen Deutschlands unterschiedlich. Während er im ostelbi- 
schen Gebiet im Verhältnis zum Westen des Landes noch relativ rückständig war, erreichten die Produktivkräfte in Südwestdeutschland bereits im 14. Jh. einen sehr hohen Stand. ${ }^{13}$

Der relative Aufschwung in der 2. Hälfte des I 5 . Jhs. resultierte nun aber in nur sehr geringem Maße aus einer Fortentwicklung der Produktionsinstrumente. Viel mehr hatte er seinen Ursprung einerseits im allmählichen Ausgleich der durch die Pestepidemien des I4. Jhs. verursachten Bevölkerungsverluste, andererseits im Úbergang zur Spezialisierung vor allem in der Viehzucht. Der ständig wachsende Fleischverbrauch der Städte und der steigende Bedarf an Rohprodukten für die gewerbliche Produktion boten für die Erzeugnisse der Viehzucht einen weitaus besseren Absatz als für Getreide. So führte der Aufschwung in der Textilproduktion z. B. zu einer bedeutenden Ausweitung der Schafzucht, insbesondere an der Mosel, in Südwestdeutschland und in Thüringen. Ebenfalls durch die Fortschritte und die daraus resultierenden Bedürfnisse der gewerblichen Produktion bedingt sind die Fortschritte in der Forstwirtschaft. Der ständig steigende Holzbedarf im Bergbau und Hüttenwesen führte zu gewaltigen Holzentnahmen, die jedoch von den Landesherren geregelt wurden: An die Stelle der ungeregelten Entnahme trat eine geregelte Schlagwirtschaft mit Einteilung der Forste in Schläge und einer planmäßigen Aufforstung.

Insgesamt zeigt sich, daß zu diesem Zeitpunkt bereits die feudale Produktionsweise nicht mehr in der Lage war, aus sich selbst heraus die Entwicklung der Produktivkräfte voranzutreiben, daß also die Motivation zu wesentlichen Verbesserungen der landwirtschaftlichen Produktion resultierten aus Bedürfnissen kapitalistisch produzierender Wirtschaftszweige. Dazu kommt, daß die feudale Abhängigkeit der Bauern die Schaffung freier Lohnarbeiter stark behinderte.

Die alte Feudalverfassung war somit auch auf landwirtschaftlichem Gebiet zu einem entscheidenden Hemmnis für den Fortschritt geworden.

\section{Soziale Struktur}

Die soeben geschilderten ökonomischen Veränderungen wirkten sich aus sowohl auf die Stellung der verschiedenen Schichten zueinander, als auch auf die Struktur dieser Schichten selbst. Um i 500 war der soziale Schichtenbau der alten Feudalgesellschaft in völliger Auflösung begriffen. Die Verschiebung in der Stellung der verschiedenen Klassen und Schichten im Produktionsprozeß führte zu einer sozialen Differenzierung, von der alle Klassen betroffen wurden. Dieser Prozeß, der sich vor allem in der Polarisierung von immer reicher werdenden Reichen und immer ärmer werdenden Armen manifestierte, trieb die sozialen Widersprüche und Spannungen auf die Spitze und schuf so eine der wesentlichen Voraussetzungen für die revolutionäre Bewegung: die gesamtnationale Krise.

Die großen Territorialfürsten hatten sich in den Besitz der meisten Hoheitsrechte gebracht und waren in ihren Ländern die Träger der politischen Gewalt. Sie besaßen in der Regel ausgedehnten Guts- und Grundbesitz und profitierten neben dem Handels- und Kreditkapital - am meisten vom ökonomischen Aufschwung.

$\mathrm{Zu}$ ihnen gehörten die Habsburger, die Wettiner (seit $\mathrm{r} 48 \mathrm{~s}$ in Albertiner und Ernestiner gespalten), die Wittelsbacher und die Hohenzollern. 
Ihr Interesse richtete sich vor allem darauf, die politische Position der Reichsgewalt zu schwächen und so selbst möglichst unabhängig zu werden.

Nach innen versuchten sie, ihre Landeshoheit weiter auszubauen und die Zentralgewalt in ihren Territorialstaaten durch Schaffung eines Verwaltungsapparates und Ausschaltung feudaler Zwischengewalten zu stärken.

Die finanziellen Mittel zur Realisierung ihrer Politik verschafften sie sich einerseits durch Erhebung feudaler Abgaben, andererseits durch Kreditnahme bei großen Handels- und Kreditfirmen, denen sie ihre Bergregalien zur Sicherheit gaben und - da sie ihre Schulden nie zurückzahlen konnten - auf diese Weise den Bergbau und den Metallhandel erheblich förderten.

Der mittlere Adel hatte als eigene Schicht fast völlig aufgehört zu existieren. Entweder hatte er es verstanden, durch geschicktes Ausnutzen der Rivalitäten unter den Territorialfürsten, die Reichsunmittelbarkeit zu erlangen, und so selbst ein kleiner Territorialherr zu werden, oder aber er war durch den Aufwand seiner Hofhaltung verschuldet und in die Reihen des niederen Adels herabgesunken. Durch die Veränderungen in Kriegswesen (Artillerie, Schießpulver, Söldnerheere) hatte der niedere Adel seine ursprüngliche feudale Kriegerfunktion ganz verloren. Infolge wachsender Konsumbedürfnisse, die durch das Angebot der Fernhändler geweckt worden waren, und der Tatsache, daß er selbst kein produktiver Stand war, sondern seine Einnahmen ausschließlich in Form von feudalen Leistungen empfing, die nicht beliebig erhöht werden konnten, war der niedere Adel zumeist völlig verschuldet und mußte im fürstlichen Hofdienst, in Landsknechtsheeren, durch Einheirat in titelsüchtiges Stadtpatriziat und durch gelegentliche Räuberei sowie vor allem durch Bauernschinderei seine Existenz sichern.

Lediglich in Südwestdeutschland konnte ein kleiner Teil seine Reichsritterschaft bewahren (Ulrich von Hutten, Franz von Sickingen etc). Diese kleine Gruppe sah naturgemäß ihren Verbündeten in der Reichsgewalt und bekämptte erbittert die geistlichen und weltlichen Fürsten.

In den Gebieten östlich der Elbe lagen die Verhältnisse anders: Die Junker, die ihre Güter selbst bewirtschafteten, konnten wegen der relativen Schwäche der Fürsten in diesem Teil Deutschlands die Polizei- und Gerichtsherrschaft in ihre Hand bekommen und so zu einer obrigkeitlichen $Z$ wischeninstanz werden. In der Folgezeit wurden sie zu großen Warenproduzenten und hatten eine enorme politische Bedeutung.

An der Spitze der städtischen Gesellschaft standen die patrizischen Geschlechter, die sog. "Ehrbarkeit «.

Ursprünglich hatte diese nur aus den reichsten Familien der Stadt bestanden, die sich auf eine althergebrachte quasi-aristokratische Stellung stützen konnten, die allein den Rat der Stadt bildeten und alle städtischen Ämter unter sich verteilten. Im Verlaufe des ökonomischen Aufschwungs nahm sie aber auch emporgekommene Elemente in sich auf. Dieses Patriziat bestand aus Grundbesitzern, Fernhändlern, reich gewordenen Zunftmeistern und Kaufleuten, beteiligte sich also zumeist sehr aktiv an frühkapitalistischen Unternehmungen und hatte somit einen bedeutenden Anteil am wirtschaftlichen Aufschwung.

Auf der anderen Seite aber beuteten sie den Großteil der Stadtbevölkerung und die zur Stadt gehörenden Bauern mittels feudaler Mechanismen bedenkenlos aus. Ja, es zeigt sich sogar ein starker Trend, die in Handel und Gewerbe erzielten Gewinne im Erwerb von Landgütern und Grundbesitz anzulegen. Insofern, als es ein entscheidendes Merkmal des Frühkapitalismus war, das ökonomische Schwergewicht vom Grundbesitz weg auf den Kapitalbesitz zu verschieben, 
war die Festlegung von Kapital in Grundbesitz und Grundherrschaft ökonomisch gesehen rückschrittlich.

Die städtischen Finanzen wurden von ihnen, die ja alle Ämter beschlagnahmt hatten, völlig willkürlich und nur zu ihrem eigenen Nutzen verwaltet. Insgesamt gesehen mußte ihnen damit jede antifeudale Bewegung als Bedrohung der eigenen Position erscheinen, weshalb sie sich denn auch zumeist mit den reaktionären Kräften verbanden.

Dem Patriziat gegenüber stand - zahlenmäßig weit überlegen (das Patriziat umfaßte selten mehr als I\% der Bevölkerung) - die bürgerliche Opposition. Sie setzte sich aus sehr verschiedenen Elementen zusammen, die aber vereint wurden durch das gleiche ökonomische und politische Interesse an der Bekämpfung des Patriziats. Von den reichen Kaufleuten und Unternehmern, die nicht dem Rat angehörten bis zu ärmeren Teilen der Handwerksmeister erstreckte sich die Skala der Mitglieder, die Engels die »gemäßigte, gesetzliche, wohlhabende und intelligente Opposition « nennt. ${ }^{14}$ Unter ihnen waren viele frühkapitalistische Unternehmer. Bei den innerstädtischen Auseinandersetzungen vor der Reformation bildeten diese bürgerlichen Mittelschichten in der Regel die Opposition. Diejenigen von ilınen, denen es gelang, größere Reichtümer anzuhäufen, verlangten politische Beteiligung, um ihre ökonomische Macht politisch verwerten zu können. Sobald sich aber das Patriziat zu geringen Kompromissen bereit zeigte, verließen sie das Lager der Opposition, um mit den reaktionären Kräften zu kollaborieren.

Der ärmere Teil der Mittelschicht kämpfte zumeist an der Seite der Plebejer, da er wie diese an einer grundsätzlichen Umgestaltung der Verfassung interessiert war.

Die plebejischen Schichten umfaßten die heruntergekommenen Elemente der alten Feudalgesellschaft und die Anfänge proletarischer Elemente, also vor allem verarmte Handwerker, Tagelöhner, Gesellen, Knechte und frühkapitalistische Lohnarbeiter. Fast völlig besitzlos und rechtslos, da sie ja kein Bürgerrecht besaßen, profitierten sie weder vom wirtschaftlichen Aufschwung, noch von den Anfängen kapitalistischer Entwicklung. Die unterste Schicht der städtischen Gesellschaft bildeten die Anfänge des Lumpenproletariats. Es waren Bettler, Gaukler, Musikanten, Reisläufer, ehemalige Landsknechte, Dirnen und Landstreicher. "In allen entwickelten Ländern war die Zahl der Vagabunden nie so groß gewesen, wie in der I. Hälfte des I6. Jhs. Ein Teil dieser Landstreicher trat in Kriegszeiten in die Armeen, ein anderer bettelte sich durchs Land, der dritte endlich suchte in den Städten durch Tagelöhnerarbeit und was sonst gerade nicht zünftig war, seine notdürftige Existenz.

Alle drei spielen eine Rolle im Bauernkrieg: der erste in den Fürstenarmeen, denen die Bauern erlagen, der zweite in den Bauernverschwörungen und Bauernhaufen, wo sein demoralisierender Einfluß jeden Augenblick hervortritt, der dritte in den Kämpfen der städtischen Parteien. «15

In der Geistlichkeit, die insgesamt ein privilegierter Stand war, gab es drei unterschiedliche Gruppen, die jeweils drei verschiedenen Klassen angehörten:

Die hohe Geistlichkeit, also die Erzbischöfe, Bischöfe, Äbte und sonstigen Prälaten, waren entweder selbst Reichsfürsten oder beherrschten als Feudalherren unter der Oberhoheit anderer Fürsten große Ländereien mit vielen Leibeigenen und Hörigen. Obgleich sie außer den gewöhnlichen Feudalabgaben und Zinsen

14 Friedrich Engels, Der deutsche Bauernkrieg, Berlin $x 970$, S. $39 \mathrm{ff}$.

15 Ders., a. a. O., S. 40. 
auch noch den Zehnten bezogen, verschafften sie sich durch die Fabrikation von wundertätigen Heiligenbildern und Reliquien und durch den Ablaßhandel noch zusätzlich gewaltige Einnahmen.

Gegen diese aristokratische Schicht innerhalb der Geistlichkeit richtete sich der Haß der gesamten Bevölkerung: der Bürger, Plebejer und Bauern, weil diese die Kosten zu tragen hatten; des Adels und der Fürsten, weil ihr flottes Wohlleben deren Neid erregte und diese hofften, sich durch Beseitigung der geistlichen Nobilität in den Besitz ihrer Reichtümer setzen zu können.

Die mittlere Geistlichkeit war zumeist akademisch und humanistisch gebildet und entstammte zum großen Teil reichen bürgerlichen Familien. Sie waren vorwiegend Anhänger gemäßigt-reformerischer Ideen und wollten vor allem selbständige Bewegungen des Volkes verhindern. Aus diesem Grund standen sie zumeist auf der reaktionären Seite.

Die niedere Geistlichkeit war vom Volkshaß ausgenommen. Sie rekrutierte sich aus den ärmeren Volksschichten und teilte deren soziale Lage und Ideen. Diese Schicht der Geistlichkeit brachte eine große Anzahl an reformatorischen und revolutionären Ideologen hervor, die in Reformation und Bauernkrieg auf der Seite der Volksmassen stritten.

Neben der Geistlichkeit, die das ganze Mittelalter hindurch Träger des Bildungsmonopols gewesen war, war - verbunden mit den ökonomisch-sozialen Veränderungen - eine von dieser verschiedene Bildungsschicht entstanden. Es handelte sich hierbei vor allem um Juristen, Lehrer, Ärzte, Professoren, Schreiber und Buchdrucker, die in fürstlichen Kanzleien, Unternehmen und Banken immer stärker Verwendung fanden.

In den Kämpfen der frühbürgerlichen Revolution findet man sie auf allen Seiten wieder.

Unter all diesen Schichten - mit Ausnahme der städtischen Plebejer - stand die große Masse der Bauern und hatte alles zu produzieren, was Fürsten, Adel, Geistliche, Patrizier und Bürger aus ihr herauspreßten.

Die ökonomische Lage der Bauern war je nach der Größe ihres Landbesitzes verschieden und relativ unabhängig davon, ob sie frei, leibeigen oder hörig waren. Gegen Ende des I s. Jhs, verschlechterte sie sich zusehends: Das Streben der Territorialfürsten nach Ausweitung und Stärkung ihrer Landeshoheit, die Funktionsbeschränkung des niederen Adels auf die Ausbeutung der Bauern, der steigende Geldbedarf des Klerus, das mit fortschreitendem ökonomischem Differenzierungsprozeß in den Städten wachsende Streben nach Aneignung des bäuerlichen Mehrproduktes durch das grundbesitzende Bürgertum und schließlich die wachsende Abhängigkeit der bäuerlichen Produzenten vom Wucherkapital - alles das ließ das Leben der Landbevölkerung unerträglich werden.

Ziel der verschärften Ausbeutung der Bauern war einmal, den Dorfgemeinschaften die Allmende, d.h. das zur Gemeinde gehörige Weideland, Moor, die Gemeinde-Wälder usw., zu nehmen und, soweit die Grundherren nicht an einer eigenen Nutzung des Gemeindelandes interessiert waren, zusätzliche Gebühren für die Nutzung durch die Dorfbewohner einzutreiben. Zum zweiten ging es darum, den Bauern das erbliche Recht auf ihren Boden zu nehmen, das die Mehrheit von ihnen in den ersten Jahrzehnten des zweiten Jahrtausends errungen hatte, und sie dafür in Zeitpächter zu verwandeln.

Außer nach einer Erhöhung der Frondienste strebten die Feudalherren gerade in Südwestdeutschland danach, die unregelmäßigen Abgaben, die im Todes- und Veräußerungsfalle zu entrichten waren, auf ein Maß zu steigern, welches einen großen Teil des beweglichen Vermögens ausmachte. Schließlich sollten die 
dieser schrankenlosen Ausbeutung unterworfenen Bauern - soweit sie noch frei waren - ihrer Freizügigkeit beraubt werden. Das alles lief auf einen Zustand hinaus, den man als zweite Leibeigenschaft bezeichnen kann.

Der wirtschaftlich und soziale Differenzierungsprozeß, der die ganze Gesellschaft erfaßt hatte, war auch an der Landbevölkerung nicht vorübergegangen.

Aus der Masse der Landbewohner hatten sich zwei Schichten abgehoben, von denen die eine eine zahlenmäßig kleine Schicht wohlhabender "Feudalbauern" war, die selbst bereits bäuerliche Arbeit ausbeutete. Ihr vorrangiges Ziel war es, ihren feudalen Besitz an Erbgütern in freies bäuerliches Eigentum umzuwandeln. Aus diesem Grunde kollidierten ihre Interessen mit denen der Feudalherrschaften, die ja bereits das bäuerliche Besitzrecht radikal einzuschränken gedachten. Obwohl die "Feudalbauern « zu ihren dörflichen Mitbewohnern in einem gewissen Gegensatz standen, wurden sie darum während der Kämpfe des Bauernkriegs Bundesgenossen der armen Bauernschaft.

Das andere Produkt des gesellschaftlichen Differenzierungsprozesses war eine große Masse landarmer oder gänzlich landloser Menschen, die aus nachgeborenen Bauernsöhnen sowie aus entlaufenen Bauern und Gesinde bestand. Sie verdingten sich zumeist als Tagelöhner in der Landwirtschaft oder auch als Lohnarbeiter in Bergbau. Ein großer Teil von ihnen schloß sich in den Kämpfen der frühbürgerlichen Revolution den Bauern an, der andere Teil ließ sich von den Söldnerheeren der Fürsten anwerben.

"Wir sehen: Die verschiedenen Stände des Reichs, Fürsten, Adel, Prälaten, Patrizier, Bürger, Plebejer und Bauern bildeten im Anfang des 16. Jhs. eine höchst verworrene Masse mit den verschiedenartigsten, sich nach allen Richtungen durchkreuzenden Bedürfnissen. Jeder Stand war dem anderen im Wege, lag mit allen anderen in einem fortgesetzten, bald offenen, bald versteckten Kampf. «16

\section{Der Charakter von Reformation und Bauernkrieg als frübbürgerliche Revolution}

Die Gesamttendenz der europäischen Entwicklung beim Übergang vom Feudalismus zum Kapitalismus verlief - in vielfältigen Misch- und Übergangsformen vom absolutistischen Zentral- zum bürgerlichen Nationalstaat. Die entscheidende Wende, die bürgerliche Revolution, fand jeweils zwischen diesen beiden Stufen statt.

Neben ihrem zwar eindeutig feudalen Charakter stellte die absolute Monarchie bei der Schaffung eines Nationalstaates und bei der Förderung der jungen kapitalistischen Elemente eine historisch notwendige, progressive Kraft dar ${ }^{17}$, insbesondere indem sie den Prozeß der ursprünglichen Akkumulation in nicht unerheblichem Maße unterstützte. So förderten die Feudalmonarchien in Spanien, Portugal, Holland, Frankreich und England die Manufakturen und den Handel durch die Gewährung von Anleihen, durch das Schutzzollsystem, durch Aufhebung der Binnenzölle und -abgaben, durch die Vereinheitlichung des Münz-, Maß- und Gewichtssystems, etc. Im internationalen Außenhandel verfochten sie die Interessen ihres nationalen Bürgertums durch Merkantilismus und Handels-

16 Ders., a. a. O., S. 43.

17 Vgl. Eckard Müller-Mertens, Zu den Aufgaben der frühbürgerlichen Revolution in Deutschland und der Rolle des Königtums, in: Tagung der Sektion Mediävistik der deutschen Historiker-Gesellschaft vom 21.-23. I. I960 in Wernigerode, Bd. II: Die frühbürgerliche Revolution in Deutschland, Berlin 196r, S. 87. 
kriege. Und schließlich benutzten sie ihre Macht dazu, die Expropriation der unmittelbaren Produzenten voranzutreiben und harte Gesetze gegen die so Expropriierten zu erlassen.

In Deutschland dagegen kam es nicht zur Entstehung eines absolutistischen Zentralstaates, sondern hier blieb die Entwicklung im Stadium der regionalen Zentralisation innerhalb der fürstlichen Territorien stecken. Bereits die Staufer hatten im Mittelalter imperiale Ziele verfolgt und die Habsburger setzten diese antinationale Politik auf anderer Ebene fort: "Die burgundische und die spanische Heirat, der Erwerb Ungarns und Böhmens, ließen ein habsburgisches Reich entstehen, in dem die Sonne nie unterging. Das deutsche Königreich war dabei bloßes Anhängsel. Es wurde zum Trittbrett degradiert. Weder Maximilian I., noch Karl V., oder Ferdinand I. traten im Sinne der progressiven Aufgabe des Königtums in Aktion. «18 Während sich also in den bezeichneten Ländern nationale Monarchien gebildet hatten, fehlte diese Vorbedingung der neuen Entwicklung in Deutschland. Aus diesem Grunde mußte das Bürgertum zusammen mit den anderen fortschrittlichen Kräften selbst die Beseitigung der Hemmnisse in Angriff nehmen, die der Fortentwicklung der Keimformen kapitalistischer Produktionsweise entgegenstanden: Im objektiven Interesse des fortschrittlichen Bürgertums wurde eine Revolution historisch notwendig.

Oben ist bereits eingehend dargestellt worden, in welchem Maße zu dieser Zeit im Schoße der feudalen Gesellschaft kapitalistische Produktionsverhältnisse entstanden und zur feudalen Produktionsweise in einen unlösbaren Widerspruch geraten waren. Entsprechend dem erst embryonalen Entwicklungsstand der kapitalistischen Elemente konnte die historische Aufgabe der Revolution noch nicht darin liegen, den Kampf um die uneingeschränkte Herrschaft der Bourgeoisie zu beginnen. Sie bestand vielmehr allgemein darin, Hindernisse, die einer raschen und umfassenden Weiterentwicklung des Kapitalismus entgegentraten, z. B. durch den Kampf um staatliche Zentralisation, den Kampf gegen die feudale Abhängigkeit der Bauern auf dem Lande, den Kampf gegen die feudalen Zunfverfassungen in den Städten, zu überwinden.

Objektiv waren somit Tendenz und Zielrichtung von Reformation und Bauernkrieg antifeudal. Der europäische Feudalismus aber konnte in keinem Lande und auch nicht in seinen einzelnen Teilen angegriffen werden, bevor nicht sein ideologisches Rückgrat, die römisch katholische Kirche, zerbrochen und eine religiöse Ideologie und kirchliche Institutionen geschaffen waren, die den objektiven Interessen des Bürgertums entsprachen. Insofern war es historisch unabdingbar, daß die Auseinandersetzung religiöse Züge trug, ja sogar in einzelnen Phasen (Reformation) auf religiösem Gebiet geführt wurde.

Die Frage nach dem Charakter eines revolutionären Ereignisses läßt sich aber nur beantworten, wenn man außer dem Grade der ökonomischen Entwicklung, dem objektiven Faktor, auch den Grad des Klassenbewußtseins und der Organisiertheit der revolutionären Kräfte, also den subjektiven Faktor, berücksichtigt. ${ }^{19}$ Die historische Triebkraft der frühbürgerlichen Revolution war das städtische Bürgertum, insbesondere dessen kapitalistisch wirtschaftende Teile. In dessen Interesse, nicht in dessen Auftrag, sollten die feudalen Hemmnisse beseitigt werden, wenngleich es sich dieses Interesses kaum oder gar nicht bewußt war. Träger der Reformation war die bürgerliche Intelligenz. ${ }^{20}$ Fast alle Reformatoren kamen aus dieser Schicht, die die Räte, Professoren, Schriftsteller und Künstler

18 Ders., a. a. O., S. 89.

19 Vgl. hierzu: W. I. Lenin, Werke Bd. 9, S. 14.

$20 \mathrm{Vgl}$. Max Steinmetz in »Beiträge«, a. a. O., S. Is4. 
stellte (z. B. Melanchthon). Träger der Hauptlast in den Kämpfen des Bauernkrieges waren die Bauern und Plebejer. Der Verlauf der Revolution wurde entscheidend beeinflußt von der Kraft und Ausdauer, die die Volksmassen während der Auseinandersetzungen entwickelten. Zu diesem Aspekt schrieb F. Engels in seiner Arbeit »Die Entwicklung des Sozialismus von der Utopie zur Wissenschaft «: »Es ist sonderbar genug: In allen drei großen bürgerlichen Revolutionen liefern die Bauern die Armee zum Schlagen, und die Bauern sind gerade die Klasse, die nach erfochtenem Sieg durch die ökonomischen Folgen dieses Sieges am sichersten ruiniert wird. $\star^{21}$

Die Erklärung dieses Phänomens mag vielleicht darin liegen, daß auf den Bauern der gesamte Schichtenbau der Feudalgesellschaft lastete, sie also zusammen mit dem Plebejertum die unterste, von allen anderen Schichten ausgebeutete Klasse bildeten. Ihre Stellung ließ sie darum vom Kampf gegen den Feudalismus subjektiv die eigene Befreiung erhoffen, wiewohl sie objektiv für die Interessen des Bürgertums kämptten.

Besonders deutlich wird diese Problematik in den sogenannten "Zwölf Artikeln«, dem zweifellos am weitesten verbreiteten Bauernprogramm während des Bauernkrieges. Eine der Hauptforderungen dieses Programms war die Aufhebung der Leibeigenschaft. Für die Bauern hätte sie subjektiv ohne Zweifel zunächst eine Befreiung bedeutet. Auf der anderen Seite aber hätte sie die massenweise Freisetzung der bäuerlichen Produzenten von Grund und Boden und deren Verwandlung in Lohnarbeiter ermöglicht und somit objektiv den Weg frei gemacht für eine weitere Entwicklung des Kapitalismus.

Wie oben bereits festgestellt, gehörte die Errichtung eines Zentralstaates zu den objektiven Kampfaufgaben der frühbürgerlichen Revolution. Zwar stellten die Bauern diese Forderung nie direkt, aber sie kämpften faktisch gegen die Träger der bestehenden Zersplitterung, gegen die Fürsten und den Feudaladel, weil deren Existenz ihre Ausbeutung begründete. Z. T. stellten sie sogar explizit die Forderung auf, daß es außer dem Kaiser keine Gewalt mehr geben dürfe. Objektiv unterstützten sie damit den Kampf um die nationale Aufgabe der frühbürgerlichen Revolution im Interesse des Bürgertums.

Insgesamt wird somit der objektiv bürgerliche Charakter von Reformation und Bauernkrieg deutlich.

Der Tatsache aber, daß sich die kapitalistischen Elemente noch in embryonalem Zustand, noch im Schoße des Feudalismus befanden, entspricht, daß es sich bei ihrem Träger, dem Bürgertum, ebenfalls erst um Keimformen der kapitalistischen Bourgeoisie handelte. Noch war es weit davon entfernt, sich als Klasse zu formieren, noch fehlten ihm alle wesentlichen integrativen Faktoren einer Klasse, insbesondere das Klassenbewußtsein und die Organisiertheit. Es fehlen somit die Voraussetzungen, die das Bürgertum zu Beginn des 16 . Jhs. hätten in die Lage versetzen können, den Kampf um die politische Macht zu beginnen, diese $\mathrm{zu}$ erobern und zu behaupten.

Insofern konnte diese im Rahmen der europäischen Entwicklung historisch notwendige Revolution nur den Charakter einen frühen bürgerlichen Revolution annehmen. Frühbürgerlich war sie aber auch in dem Sinne, als durch sie der Prozeß der bürgerlichen Revolutionen in ganz Europa eingeleitet wurde: "Auf Luther folgt Zwingli, auf Zwingli Calvin «22, Reformation und Bauernkrieg sind

21 Fredrıch Engels, a. a. O., in: Karl Marx und Friedridh Engels, Ausgewählte Werke, Moskau I 971 , S. 406.

22 Max Steinmetz, Ober den Charakter der Reformation und des Bauernkrieges in Deutschland, in: R. Woblfeil (Hrsg.), Reformation oder frühbürgerlidhe Revolution, Mündien 1972, S. Is3. 
die erste Stufe auf der Stufenleiter bürgerlicher Revolutionen im europäischen Maßstab, deren zweite die Englische und deren Höhepunkt die Französische Revolution ist, weil sie als erste bis zur Vernichtung des Feudalismus und dem endgültigen Sieg der Bourgeoisie ausgekämpft wurde.

Zur Situation des Rechts in Deutschland zu Beginn des 16. Jbs.

Die Intensivierung der Ware - Geld - Beziehungen, das Aufkommen von Frühformen kapitalistischer Produktionsweise, der quer durch alle Schichten gehende soziale Differenzierungsprozeß, und schließlich die auf die Zuspitzung zur gesamtnationalen Krise folgende frühbürgerliche Revolution waren begleitet von einschneidenden Veränderungen im juristischen Überbau.

Besonders hinderlich für die ökonomisch-soziale Entwicklung Deutschlands war die feudale Rechtszersplitterung und die fehlende wissenschaftliche Bearbeitung des Rechts. Die sich ständig verkomplizierenden Rechtsfälle forderten exakte Rechtsbegriffe und allgemeine Gesetze, da die in Weistümern aufgezeichneten Präzedenzfälle nicht mehr für die schwierigen Konfliktslagen einer sich entfaltenden Warenwirtschaft ausreichten. Einerseits warf das »arbeitende« Kapital Rechtsfragen auf, die das alte deutsche Recht nicht gekannt hatte, andererseits führte die leichte Beweglichkeit von Geld und Kapital in größerem Maße und in anderer Weise zu Straffälligkeiten, als zuvor in der feudalen Naturalwirtschaft. Auch in der Gerichtsverfassung und im Prozeßrecht trat die Rückständigkeit der alten Regelungen nunmehr offen zutage. "Die Schöffenjustiz versagte vor den Problemen des Frühkapitalismus. «23

In der Mitte des 14. Jhs. hatte in Deutschland die sog. Rezeption des römischen Rechts begonnen, ein Prozeß der Übernahme römisch-italienischer Rechtsformen und Regelungsmodelle. Römisches Recht war zwar bereits im Mittelalter durch die sogenannte "Vorrezeption « im II. Jh. in Deutschland bekannt gewesen, von einer Rezeption im eigentlichen Sinne kann aber erst im ausgehenden i 4. Jh. gesprochen werden, als deutsche, vorwiegend bürgerliche, Studenten an oberitalienischen Universitäten römisches Recht in einem nach scholastischer Methode entworfenen System studierten, wie es die Postglossatoren (v.a. Bartolus und Baldus) im I3. und 14. Jh., also der Blütezeit des italienischen Handelskapitalismus (Venedig, Genua, Pisa, Neapel), entwickelt hatten.

Uber die Gründe, warum bereits im späten 14. Jh. deutsche Studenten in Oberitalien profanes römisches Recht studierten, läßt sich aufgrund des vorhandenen Materials noch keine fundierte Aussage machen. Wieackers ${ }^{24}$ Begründung, daß zu dieser Zeit bereits in den Verwaltungen der weltlichen und geistlichen Fürsten sowie in den Stadtverwaltungen Bedarf nach Juristen bestanden habe, kann nicht befriedigen, da sie das Faktum des Bedarfs seinerseits unhinterfragt läßt.

In den Jahrzehnten um I 500 jedenfalls, als sich in Deutschland eine entfaltete Warenwirtschaft entwickelt hatte und bereits Keimformen kapitalistischer Produktionsweise existierten, trat der oben erwähnte Rezeptionsvorgang in seine Endphase ein und wurde erst jetzt yoll wirksam, als römische Rechtsformen Eingang fanden in die Rechtsprechung und Verwaltung der Territorialfürsten.

Warum gerade das römische Recht dazu ausersehen wurde, die Regelungsmuster und Ordnungsmechanismen für die neue sozio-ökonomische Struktur (nicht nur

23 Mitteis-Lieberich, a. a. O., S. I91.

24 Franz Wieacker, a. a. O., S. Is2 ff. 
in Deutschland, sondern - außer in England und der Schweiz - im gesamten Mitteleuropa) zu liefern, wird deutlich, wenn man sich die Interessen- und Bedürfnislage der zwei entscheidenden Kräfte in dieser Zeit, der Territorialfürsten und des kapitalistisch wirtschaftenden Bürgertums, vor Augen führt: Vorrangiges Ziel der Fürsten war der Ausbau ihrer Landeshoheit und die Errichtung absolutistischer Zentralstaaten, in denen sie über die Angehörigen aller Stände als Untertanen herrschen konnten. Das Interesse des Bürgertums verlangte ein Recht, das den Erfordernissen der Warenproduktion entsprach, das folglich die Rechtsverhältnisse zwischen Personen als unpersönliche, durch die Institution des Marktes vermittelte, begreifen mußte. Beide Interessenlagen waren somit Ausdruck ihrer historischen Situation: des beginnenden Uberganges von einer feudalen Gesellschaftsordnung, die idealtypisch von Naturalwirtschaft und persönlichen Bindungen geprägt war, zur bürgerlich-kapitalistischen, die geprägt ist von Geldwirtschaft und institutionellen Bindungen. ${ }^{25}$ Römisches Recht war vollendetes Recht der einfachen, also vorkapitalistischen Warenproduktion. ${ }^{26}$ Es entsprach somit den ökonomischen Verhältnissen, die im Mittelalter durch die Teilung der Arbeit zwischen Stadt und Land entstanden waren. Außerdem war es insofern sehr entwicklungsfähig, als die meisten Rechtsverhältnisse der kapitalistischen Produktion durch die der einfachen Warenproduktion erfaßt werden. Römisches Recht war darüber hinaus der beispielhafte juristische Ausdruck einer Gesellschaft, in der das reine Privateigentum herrscht. Es war darum dem bürgerlichen Eigentum des ausgehenden Mittelalters weit voraus, das noch stark mit feudalen Bindungen, zumindest soweit es in Privilegien bestand, behaftet war. Da sich das bürgerliche Eigentum nun historisch notwendigerweise zum reinen Privateigentum weiterentwickeln mußte, war das römische Recht ein enormer Motor dieser Entwicklung. ${ }^{27}$

Wir hatten oben gesehen, daß die kapitalistische Produktionsweise und dementsprechend auch das kapitalistisch wirtschaftende Bürgertum zwar die progressive Kraft in dieser Zeit darstellte, daß es aber gleichwohl erst in Keimformen existierte, insbesondere an der politischen Macht kaum partizipierte. Die eigentliche Gewalt lag vielmehr bei den Territorialfürsten.

Das neue Recht mußte also auch und gerade den Bedürfnissen der Zentralgewalten genügen, um Eingang in alle wesentlichen Bereiche der Territorialstaaten finden zu können. Das römische Recht war das einer Sklavenhaltergesellschaft gewesen. Seine Rechtsnornen behandelten darum alle Rechtssubjekte im wesentlichen als Gleiche, da die Sklaven nur Rechtsobjekte gewesen waren. Insofern boten die römischen Rechtsquellen eine ausgezeichnete Grundlage zum Ausbau der Landeshoheit in Richtung auf den Absolutismus, der schließlich ebenfalls alle Rechtssubjekte relativ gleich als Untertanen behandelte. "Die römischen Rechtsquellen waren eine dem Willen der herrschenden Klasse im wesentlichen adaequate Form. Zugleich entsprachen sie auch den bürgerlichen Gleichheitsvorstellungen. Die Verwendung der ihrem Charakter nach antifeudalen Rechtsformen war daher ein fortschrittlicher Akt, auch wenn er durch die feudale Reaktion letztlich veranlaßt wurde. Eine Ablehnung der Rezeption, besser, der Ver-

$25 \mathrm{Vgl}$. hierzu: K. H. Blaschke, Frühkapitalismus und Verfassungsgeschichte, in: Wiss. Zeitschrift der Karl-Marx-Universität, Leipzıg 1965, S. $435 \mathrm{ff}$.

20 Vg1. Gerbard Günther, Altes Recht, Göttliches Recht und Römisches Recht in der Zeit der Reformation und des Bauernkrieges, in: Wiss. Zeitschrift der Karl-Marx-Universität, Leıpzig I 965, S. $427 \mathrm{ff}$.

$27 \mathrm{Vgl}$. Friedrich Engels, Ober den Verfall des Feudalismus und das Aufkommen der Bourgeoisie, in: MEW, Bd. 21, S. 397. 
such, sie rückgängig zu machen, (wie das der Faudaladel, die Bauern und die

Plebejer angestrebt hatten, Anm.d. Verf.) war daher Maschinenstürmerei auf ideologischem Gebiet. «28

Das kaiserliche Recht, das die Juristen auf den Universitäten als Justinianisches gelernt und ursprünglich im Dienste des Kaisers angewandt hatten, verdrängte in zunehmendem Maße das kanonische Recht. In Verbindung mit dem im Mittelalter neben dem kanonischen gültigen deutschen Recht bildete sich allmählich aus römischem, kanonischem und deutschem Recht ein Mischrecht heraus, das sogenannte "Gemeine Recht «. Für dessen Anwendung galt zunächst die »salvatorische Klausel «, die besagte, daß es nur subsidiär eingreife hinter bewiesenermaßen vernünttigem deutschem Recht. Indem aber die Anforderungen an diesen Beweis ständig hochgeschraubt wurden, verkehrte sich diese Regel allmählich in ihr Gegenteil. ${ }^{20}$

Seit dem is. Jh. entstanden massenhaft neue Partikularrechte, also Stadt- und Landrechte, auf der Grundlage der rezipierten Rechtsquellen. Die erste Welle dieser Neubildungen vollzog sich in den Städten, als sogenannte Stadtrechtsreformationen, und zwar in Nürnberg I 479, Worms 1498, Frankfurt a. M. I s09, Freiburg im Breisgau i 520 . Neue Territorialrechte bildeten sich i 5 I 8 in Bayern, I 27 in Brandenburg, Is 55 in Württemberg, IS7 In Solms und IS72 in Kursachsen.

Die Reichsgewalt versuchte 1495 mit der Ordnung des Reichskammergerichts und I 32 mit der Peinlichen Gerichtsordnung Karls V. (Constitutio criminalis carolina, CCC), deren Vorbild die Bambergische Halsgerichtsordnung von i 507 war, die Rechtsentwicklung in die gleiche Richtung zu lenken. Außer in der Schaffung allgemeiner Notariatsordnungen (ISI2) wurde ihr Eingreifen vor allem notwendig im Zuge der Geldentwertung, die in erster Linie durch die von den Territorialfürsten betriebene Münzverschlechterung verursacht wurde. Mittels Polizeiverordnungen (I 530, I 548, I 577 ) und der Münzordnung von i 559 versuchte sie, der Lage Herr zu werden.

Die ökonomische Entwicklung machte aber auch die rechtliche Fixierung völlig neuer Gebiete notwendig. Aus diesem Grunde kam es zur Herausbildung von Bergrecht, Transportrecht, Schiffahrtsrecht, Gewerberecht und Druckrecht. Diese Rechtsmaterien blieben, ebenso wie das Handelsrecht, vom römischen Recht unberührt. ${ }^{30}$ Der Grund dafür ist nun aber nicht, daß das römische Recht hier etwa keine Vorbilder geboten hätte. (Diese Lücke hätten die Juristen durch noch so kühne Analogien leicht ausgefüllt, wie sie das ja auch auf anderen Gebieten getan haben und tun). Vielmehr hatte das Bürgertum auf diesen Gebieten mittlerweile eine so führende Position inne, daß sich hier bereits ein eigener juristischer Ưberbau bilden konnte. ${ }^{31}$

Wie oben bereits festgestellt wurde, war dem Klerus durch die Buchdruckerei und die Bedürfnisse des ausgedehnteren Handels das Monopol nicht nur des Lesens und Schreibens, sondern auch der höheren Bildung genommen worden. Insbesondere entstand im Zusammenhang mit der Rezeption eine eigene, von vornherein fast ausschließlich bürgerliche Juristenschicht, so daß die Träger von Theologie und Jurisprudenz von nun an nicht mehr identisch waren. Der neue

\footnotetext{
28 Gerhard Günther, a. a. O., S. 432.

29 Mitteis-Lieberich, a. a. O., S. 192.

30 Ders., a. a. O., S. 192.

31 Vgl. hierzu: Heınz Herz, Die Rezeptıon des römischen Rechts im Blidkfeld des historischen Materialismus, in: Joh. Irmscher (Hrsg.), Renasssance und Humanısmus in Mittel- und Osteuropa, Berlin 1962 , S. $38 \mathrm{ff}$.
} 
Stand verdrängte die Geistlichkeit nahezu vollständig aus den Amtern der Justiz, der Verwaltung und sogar der Rechtswissenschaft, soweit sie profanes Recht betraf. Die professoralen Exponenten waren oft Lehrer, Gutachter und angestellte Rechtskonsultenten in Personalunion. Selbst bischöfliche Kanzleitätigkeit und städtisches Amt waren für Zasius, einen der hervorragendsten Rechtsgelehrten dieser Zeit, durchaus vereinbar. Die bürgerlichen Juristen waren allfunktionell. Es zeigte sich bei ihnen die zunehmende Tendenz, ihre Tätigkeit als Ware zu begreifen. So meinte zum Beispiel Alciat, daß die Höhe der Gutachterpreise und Professorengehälter indizierten, daß die Rechtswissenschaft auf der Höhe sei. ${ }^{32}$ Im Bereich der Justiz kam es zu Installierung fester Gerichtshöfe, wobei das Gerichtswesen nichtsdestoweniger unübersichtlich blieb, da die Gerichtsherren überall nach Erweiterung ihrer Kompetenzen strebten. An die Stelle von Laienschöffen und -richtern - auch der Landesherr war in diesem Sinne ein Laie traten gelehrte Juristen, unter denen anfangs das adlige Element kaum vertreten war.

Die aufsteigenden Territorialgewalten hatten dem Reich zuerst die niedere Gerichtsbarkeit, später auch die Hochgerichtsbarkeit entrissen. Es entwickelte sich ein landesherrlicher Instanzenzug: Adel, höhere Geistlichkeit und Städte suchten Recht bei den ehemaligen Hochgerichten ohne Rücksicht auf den Gegenstand der Klage. Für die Bauern blieb fast ausschließlich die niedere Gerichtsbarkeit zuständig, die in der Form von Dorf- oder Kirchspielgerichten unter Vorsitz des Dorfschulzen tagte. Auch die Verpfändung von Gerichtsherrschaften war an der Tagesordnung, so daß Bürgerliche und Adlige die Befugnis erwerben konnten, die Richter einzusetzen. ${ }^{33}$

Außerhalb der nunmehr staatlichen Sphäre bildeten sich ebenfalls Gerichte heraus: Der jeweilige Lehensherr führte den Vorsitz in den Lehensgerichten, Grundherren urteilten über die von ihnen abhängigen Bauern, für Bergrechtsfragen waren Berggerichte zuständig und die kirchliche Gerichtsbarkeit lag in den Händen eines vom Bischof bestellten Offizials.

Das neue Recht, das im Gegensatz zur alten Schöffenjustiz ein schrifliches Recht war und - wie wir gesehen haben - gelehrte Juristen und Advokaten voraussetzte, brachte weder dem Feudaladel noch den Bauern und Plebejern irgendeinen Vorteil. Die Rechtsprechung hatte sich nur verteuert und widersprach der in großen Zeiträumen entstandenen Rechtstradition und dem Rechtsempfinden des Volkes. Juristen, Advokaten und Gerichtsschreiber wurden in der Vorstellungswelt dieser Schichten zu Vertretern des Unrechts. (» Juristen sind böse Christen «, so lautete ein Sprichwort in dieser Zeit.)

Der Verlust einer einheitlichen Trägerschicht und einer einheitlichen Rechtsquelle ist begleitet vom Verlust einer einheitlichen wissenschaftlichen Methode. Das seit dem 16 . Jh. in Deutschland angewandte und gelehrte Recht zerfiel in zwei Hauptströmungen, bzw. Literaturkreise, die sich $\mathrm{z}$. T. überschnitten und in vielen Mischformen begegneten:

Die scholastische Dialektik hatte versucht, die Autoritäten der Religion (hl. Schrift) und der Philosophie (Aristoteles) in ihrer Übereinstimmung zu erkennen und zu beweisen. Ihre Prämisse war, daß die Wahrheit gegeben und nicht zu finden, sondern aus der Überlieferung zu lernen sei. Ihr Ziel war es, die Übereinstimmung metaphysischer Wahrheiten, welche als Inhalt der menschlichen

\footnotetext{
32 Vgl.: ZRG 1970: Rezension eines Bandes der Amerbach-Korrespondenz: Alciat feilscht um einen Gutachterpreis.

33 Vgl.: Max Stetnmetz, Betträge, a. a. O., S. 6x.
} 
Vernunft erschienen, mit der Offenbarung aufzuzeigen. Ihr Ziel war es zum anderen, den Inhalt beider auf dem Wege der Analyse zur Erkenntnis zu bringen. Analyse bedeutete hier eine Methode, die den Inhalt als einen gegebenen und fertigen zugrunde legte und ihn durch syllogistische Beweisführung zergliederte.

Die oberitalienische Jurisprudenz hatte sich an dieser Methode orientiert. Auch sie wollte keine neuen Wahrheiten finden, sondern objektiv gegebene Sätze ermitteln. Dazu war ihre Argumentationsweise exegetisch und am verbindlichen Text orientiert. Prinzipiell war sie abhängig von der Autorität der gelehrten Tradition. Ihr Gegenstand war das überlieferte Recht in seiner zeitgenössischen Geltung. Im Laufe der Zeit bildete sich ein fester Typus dieser analytisch-exegetischen Methode heraus, der seit dem I6. Jh. - bereits in Abgrenzung zu anderen Methoden - "mos italicus" genannt wurde. Diese Methode - auch »mos magistralis « genannt - war anfangs in Deutschland allgemein üblich und wurde später durch Statuten gegen andere Verfahrensweisen abgesichert.

Unter dem Einfluß des Humanismus entstand - ebenfalls im Ưbergang zum I6. Jh. - eine Rechtswissenschaft, die ihre Impulse von der Literatur des Quattrocento empfangen und somit Berührung hatte mit allen sozialen und ideologischen Phänomenen dieses Zeitalters. Wie der mos italicus war sie aus der mittelalterlichen Kommentatorenjurisprudenz hervorgegangen und argumentierte ebenfalls exegetisch und auf derselben verbindlichen Quellenbasis. Im Gegensatz zu ihm löste sie sich aber vom scholastischen Interpretationsschema und behandelte die Texte historisch-kritisch. Sie wollte römisches Recht aus seiner spezifisch historischen Situation heraus interpretieren, d. h. die römischen Texte waren so zu lesen, wie sie zur Zeit ihrer Entstehung gemeint waren. Die historischsystematische Methode war in Frankreich entstanden und hieß deshalb "mos gallicus«. Sie war humanistische Jurisprudenz und versuchte, durch Textkritik am Corpus juris, durch intensive Studien der Dialektik und Logik (diese waren allerdings kein Spezifkum humanistischer Rechtswissenschaft, sondern humanistisches Allgemeingut) ein neues, in sich einheitliches Rechtssystem zu schaffen, einen ordo juris, der die Grundlage für eine neue Theorie und Praxis juristischen Argumentierens abgeben könnte. Juristische Entscheidungen sollten nicht mehr im Ergebnis willkürlich, und in der Begründung autoritär sein, sondern man hoffte, aus einem einheitlichen, widerspruchsfreien Rechtssystem kalkulierbare, d. h. bei gleicher Konfliktslage gleichlautende Urteile logisch $z$ wingend ableiten zu können. Diese Logik- und Dialektikstudien des r6. Jhs. waren, wie H.E. Troje mit Recht ausführte, "Ausdruck der Auflösung der mittelalterlichen klerikalfeudalen Ständegesellschaft und des Zerfalls der Einheit ihrer Ideologie (oder, anders gefaßt: Ausdruck des Ưberganges von zünftig-feudalistischer zu kapitalistischer Produktionsweise). Alle Tendenzen, in juristischen Methodenlehren autoritäre Begründungsweisen zugunsten rationaler einzuschränken, verraten, daß die Jurisprudenz ihre Funktion, politische Entscheidungen zu motivieren, allein mit der Berufung auf Autorität, Tradition und Vorurteil und dem bloßen Schein einer dialektischen Schulung nicht länger erfüllen konnte. Die mit Einsatz von Instrumenten der neuen »Dialektik " (jetzt oft synonym mit Logik und Topik) unternommenen Versuche des Neubaus eines Gesamtsystems juristischer Dogmatik sind von der Hoffnung getragen, die verlorene Einheit der Gesellschaft lasse sich im Bemühen um rationale gesellschaftliche Ordnungen zurückgewinnen. Diese Hoffnung wiederum ist getragen von der humanistischen (letztlich stoischen) Illusion einer Koinzidenz von ratio und natura, von Gedanke und Wirklichkeit, von rationaler Ordnungsmethode und konkreter politischer Macht- 
148 ausübung. So antworten alle Bemühungen um einen rationalen ordo juris, (wie mutatis mutandis - die Systemideologien des r 9 . Jahrhunderts) in mehrfacher Hinsicht den Notwendigkeiten des kapitalistischen Warenverkehrs: Durch Rechtssicherheit (certitudo juris) im Sinne von Voraussehbarkeit, Kalkulierbarkeit des Rechts wird dieser zuallererst ermöglicht « ${ }^{34}$

34 Hans Erich Troje, Manuskript, Frankfurt/Main 1972. 